\title{
ON THE FACTORIZATION OF INTEGRAL FUNCTIONS WITH $p$-ADIC COEFFICIENTS.
}

\author{
BY PROFESSOR L. E. DICKSON.
}

(Read before the American Mathematical Society, September 6, 1910.)

1. IF $F(x)$ is an integral function of degree $n$ with integral $p$-adic coefficients, then for any integer $k$ we have a congruence

$$
\begin{aligned}
F(x) \equiv F^{(k)}(x) & =F_{0}(x)+p F_{1}(x)+p^{2} F_{2}(x) \\
& +\cdots+p^{k} F_{k}(x) \quad\left(\bmod p^{k+1}\right),
\end{aligned}
$$

in which each $F_{i}(x)$ is an integral function of degree $\leqq n$ with coefficients belonging to the set $0,1, \ldots, p-1$. The function $F^{(k)}(x)$ is called the convergent of rank $k$ of $F(x)$. If

$$
F(x)=f(x) \cdot g(x)
$$

in which the factors are integral functions with integral $p$-adic coefficients, then for any integer $k$ we obviously have

$$
F^{(k)}(x) \equiv f^{(k)}(x) \cdot g^{(k)}(x) \quad\left(\bmod p^{k+1}\right) .
$$

The following converse theorem plays a fundamental rôle in Hensel's new theory of algebraic numbers :* If

$$
F(x) \equiv f_{0}(x) \cdot g_{0}(x)
$$

for $\dagger s+1>2 \rho$, where $\rho$ is the order of the resultant $R$ of $f_{0}(x)$ and $g_{0}(x)$, then $F(x)$ is the product $(2)$ of two integral functions with integral $p$-adic coefficients whose convergents of rank $s-\rho$ are $f_{0}(x)$ and $g_{0}(x)$.

Hensel's proof is in effect a process to construct the successive convergents of $f(x)$ and $g(x)$. Each step of the process requires the solution of a linear equation in two unknowns with $p$-adic coefficients. The object of this note is to furnish a decidedly simpler process, which dispenses with these linear 71.

* Hensel, Theorie der algebraischen Zahlen, Leipzig, Teubner, 1908, p.

† This condition is satisfied if $s=\delta$, where $\delta$ is the order of the discriminant of $F(x)$. Hence we obtain as a corollary the theorem of Hensel, page 68. 
equations, and requires only the solution of a single linear congruence.

By the remark of Hensel, bottom of page 64, we may assume that the leading coefficients in $F(x), f_{0}(x)$, and $g_{0}(x)$ are powers of $p$, so that $F-f_{0} g_{0}$ is of degree less than $n$.

2. For the sake of simplicity, we first establish the theorem for the important case $s=\rho=0$ : If $F(x)=F_{0}(x)+p F_{1}(x)$ $+\cdots$, in which the coefficient of $x^{n}$ in $F_{0}$ is unity and $F_{i}(i>0)$ is of degree less than $n$, and if

$$
F_{0}(x) \equiv f_{0}(x) \cdot g_{0}(x)
$$

in which $f_{0}$ and $g_{0}$ are integral functions of degrees $\mu$ and $\nu$ respectively, with integral coefficients, while $f_{0}$ and $g_{0}$ have no common factor modulo $p$, then integral functions $f_{i}(x)$ of degrees $<\mu$ and $g_{i}(x)$ of degrees < $\nu$ with integral coefficients can be so determined that

$$
f=f_{0}+p f_{1}+p^{2} f_{2}+\cdots, \quad g=g_{0}+p g_{1}+p^{2} g_{2}+\cdots
$$

have as their product $F(x)$. It is meant by the last statement that congruence (3) holds for every integer $k$.

Since $f_{0}$ and $g_{0}$ have no common factor, integral functions $a(x)$ and $b(x)$ with integral coefficients can be determined (for example, by Euclid's process) such that

$$
a f_{0}+b g_{0} \equiv 1
$$

By (5), $F_{0}-f_{0} g_{0}$ is the product of $p$ by an integral function with integral coefficients which may be designated $L_{1}(x)-F_{1}(x)$,

$$
F_{0}-f_{0} g_{0}=p\left(L_{1}-F_{1}\right) \text {. }
$$

By the remark at the end of $\S 1, L_{1}$ like $F_{1}$ is of degree less than $n$. The congruence

$$
F_{0}+p F_{1} \equiv\left(f_{0}+p f_{1}\right)\left(g_{0}+p g_{1}\right)
$$

is equivalent, in view of $(7)$, to

$$
L_{1} \equiv f_{0} g_{1}+g_{0} f_{1}
$$

In view of (6), every set of solutions is given by

$$
f_{1} \equiv b L_{1}-\rho_{1} f_{0}, \quad g_{1} \equiv a L_{1}+\rho_{1} g_{0} .
$$

We choose $\rho_{1}(x)$ so that the degree of $f_{1}$ shall be less than the degree $\mu$ of $f_{0}$. Then the final term of (8) is of degree $<\mu+\nu$, 
so that the degree of $g_{1}$ is $<\nu$. Hence the required functions $f_{1}$ and $g_{1}$ are given by (9).

To make the general step by induction, suppose that, for $i=1, \cdots, k$, functions $f_{i}$ of degrees $<\mu$ and $g_{i}$ of degrees $<\nu$ have been determined so that (3) holds. Hence we may set

$$
F^{(k)}=f^{(k)} g^{(k)}+p^{k+1}\left(L_{k+1}-F_{k+1}^{\prime}\right),
$$

where $L_{k+1}$ is of degree less than $n$. Since

$$
\begin{gathered}
F^{(k+1)}=F^{(k)}+p^{k+1} F_{k+1}, \quad f^{(k+1)}=f^{(k)}+p^{k+1} f_{k+1}, \\
g^{(k+1)}=g^{(k)}+p^{k+1} g_{k+1},
\end{gathered}
$$

the condition for the congruence

$$
F^{(k+1)} \equiv f^{(k+1)} g^{(k+\mathrm{i})}
$$

becomes, upon applying (10),

$$
L_{k+1} \equiv f^{(k)} g_{k+1}+g^{(k)} f_{k+1} \equiv f_{0} g_{k+1}+g_{0} f_{k+1} \quad(\bmod p) .
$$

The general set of solutions is

$$
f_{k+1} \equiv b L_{k+1}-\rho_{k+1} f_{0}, \quad g_{k+1} \equiv a L_{k+1}+\rho_{k+1} g_{0} .
$$

We determine $\rho_{k+1}$ so that the degree of $f_{k+1}$ shall be less than the degree $\mu$ of $f_{0}$; then the degree of $g_{k+1}$ will be less than the degree $\nu$ of $g_{0}$. Since the induction is complete, our theorem is proved.

3. We readily deduce recursion formulas for the functions $L_{i}$. We have proved that functions $L_{i}$ and $\rho_{i}$ can be determined so that

$$
f_{i}=b L_{i}-\rho_{i} f_{0}, \quad g_{i}=a L_{i}+\rho_{i} g_{0}
$$

give functions $f_{i}$ of degrees $<\mu$ and $g_{i}$ of degrees $<\nu$ for which congruence (3) holds for every $k$. From (6),

$$
a f_{0}+b g_{0}=1+p \lambda(x) \text {. }
$$

In $(10)$ we replace $F^{(k)}, f^{(k)}, g^{(k)}$ by the values obtained by replacing $k$ by $k-1$ in (11) and then replace $F^{(k-1)}$ by the value obtained by replacing $k$ by $k-1$ in (10). After deleting the factor $p^{k}$, we get

$$
L_{k}=f_{k} g^{(k-1)}+g_{k} f^{(k-1)}+p^{k} f_{k} g_{k}+p L_{k+1}-p F_{k+1} \text {. }
$$

In the terms $f_{k} g_{0}+g_{k} f_{0}$ we replace $f_{k}$ and $g_{k}$ by their values 
(12) and apply (13). After deleting the factor $p$ we get

(14) $L_{k+1}=F_{k+1}-\lambda L_{k}-f_{k_{k}}[g]_{k-1}-g_{k}[f]_{k-1}-p^{k-1} f_{l_{k}} g_{k}$,

in which we have employed the abbreviation

$$
[z]_{i}=z_{1}+p z_{2}+p^{2} z_{3}+\cdots+p^{i-1} z_{i}, \quad[z]_{0}=0 .
$$

If in (10) we set

$$
\begin{gathered}
F^{(k)}=F_{0}+p[F]_{l}, \quad f^{(k)}=f_{0}+p[f]_{k}, \quad g^{(k)}=g_{0}+p[g]_{l k}, \\
\gamma=\left(F_{0}-f_{0} g_{0}\right) / p,
\end{gathered}
$$

and then delete the factor $p$, we get

$f_{0}[g]_{k}+g_{0}[f]_{k}+p^{k} L_{k+1}=\gamma+[F]_{k}+p^{k} F_{k+1}-p[f]_{k}[g]_{k}$. By (12), (15), the sum of the first two terms equals

$$
\sum_{i=1}^{l_{k}} p^{i-1}\left(a f_{0}+b g_{0}\right) L_{i}=(1+p \lambda) \sum_{i=1}^{k} p^{i-1} L_{i}=(1+p \lambda)[L]_{l i} \text {. }
$$

Hence we obtain the formula

$$
[L]_{k+1}=\gamma+\left[F^{\prime}\right]_{k+1}-p \lambda[L]_{k}-p[f]_{k}[g]_{k} .
$$

It is also easy to establish this formula by induction.

4. To prove the more general theorem of $\S 1$, we apply the method of $\S 2$ with congruence (6) replaced by

$$
a f_{0}+b g_{0} \equiv p^{\rho} \quad\left(\bmod p^{s+1}\right) .
$$

Since the resultant of $f_{0}(x)$ and $g_{0}(x)$ is divisible by $p^{\rho}$, but by no higher power of $p$, solutions $a(x)$ and $b(x)$ of $(17)$ can be determined by the method given by Hensel on pages 62,63 . In view of (4),

$$
F^{(s+1)}-f_{0} g_{0}=p^{s+1} L_{1},
$$

where $L_{1}$ is of degree less than $n$. Then the congruence

$$
F^{(s+1)} \equiv\left(f_{0}+p f_{1}\right)\left(g_{0}+p g_{1}\right) \quad\left(\bmod p^{s+2}\right)
$$

is satisfied if we take

$$
f_{1} \equiv p^{s-\rho}\left(b L_{1}-\rho_{1} f_{0}\right), \quad g_{1} \equiv p^{s-\rho}\left(a L_{1}+\rho_{1} g_{0}\right) \quad\left(\bmod p^{s+1}\right),
$$

since by (17)

$$
f_{0} g_{1}+f_{1} g_{0} \equiv p^{s} L_{1} \quad\left(\bmod p^{s+1}\right)
$$


and $p^{2} f_{1} g_{1}$ is divisible by $p^{t}$, where $t=2+2 s-2 \rho>s+1$. We choose $\rho_{1}(x)$ so that the degree of $f_{1}(x)$ shall be less than the degree of $f_{0}(x)$; then by (20) the degree of $g_{1}(x)$ will be less than the degree of $g_{0}(x)$.

Similarly, if in accord with (18) we set

$$
F^{(s+1)}-\left(f_{0}+p f_{1}\right)\left(g_{0}+p g_{1}\right)=p^{s+2}\left(L_{2}-F{ }+2\right),
$$

the congruence

$$
F^{(s+2)} \equiv\left(f_{0}+p f_{1}+p^{2} f_{2}\right)\left(g_{0}+p g_{1}+p^{2} g_{2}\right) \quad\left(\bmod p^{s+3}\right)
$$

is satisfied if we take

$$
f_{2} \equiv p^{s-\rho}\left(b L_{2}-\rho_{2} f_{0}\right), \quad g_{2} \equiv p^{s-\rho}\left(a L_{2}+\rho_{2} g_{0}\right) \quad\left(\bmod p^{s+1}\right) .
$$

The general step in the proof may now be made as in $\S 2$.

\section{HENSEL'S THEORY OF ALGEBRAIC NUMBERS.}

Theorie der Algebraischen Zahlen. Von Kunt Hensel.

Erster Band. Leipzig and Berlin, Teubner, 1908. xi + $346 \mathrm{pp}$.

In the theory of functions one may investigate an analytic function in the neighborhood of a point $z=a$ by means of a power series in $z-a$. In arithmetic one usually employs only developments according to the fixed base 10. The author undertakes in the present work to introduce a corresponding mobility into arithmetic and algebra by employing expansions of numbers into power series in an arbitrary prime number $p$.

A positive integer $D$ can be expressed in one and but one way in the form

$$
D=d_{0}+d_{1} p+\cdots+d_{k} p^{k}
$$

in which each $d_{i}$ is one of the integers $0,1, \ldots, p-1$. This equation will be said to define the representation of $D$ as a $p$ adic number, for which the following symbol will be employed:

For example,

$$
D=d_{0}, d_{1} d_{2} \cdots d_{t_{c}}(p) \text {. }
$$

$$
14=2+3+3^{2}=2,11(3), \quad 216=2 \cdot 3^{3}+2 \cdot 3^{4}=0,0022
$$

The sum of two such $p$-adic numbers is readily expressed as a $p$-adic number. For example,

$$
0,0022+0,1021=0,10111
$$

